\title{
Modification of Clays by Sol-Gel Reaction and Their Use in the Ethylene In Situ Polymerization for Obtaining Nanocomposites
}

\author{
E. Moncada, ${ }^{1}$ R. Quijada, ${ }^{2,3}$ and P. Zapata ${ }^{4}$ \\ ${ }^{1}$ Grupo de Investigación en Materiales Avanzados y Energía, Centro de Investigaciones, Instituto Tecnológico Metropolitano, \\ A.A 54959 Medellín, Colombia \\ ${ }^{2}$ Departamento de Ingeniería Química, Facultad de Ciencias Físicas y Matemáticas, Universidad de Chile, Santiago, Chile \\ ${ }^{3}$ Center for Advanced Interdisciplinary Research in Materials (CIMAT), Santiago, Chile \\ ${ }^{4}$ Departamento de Química y Biología, Universidad Santiago de Chile, Santiago, Chile
}

Correspondence should be addressed to E. Moncada, edwinmoncada@itm.edu.co

Received 24 April 2011; Revised 11 August 2011; Accepted 30 August 2011

Academic Editor: Amir Kajbafvala

Copyright ( 92012 E. Moncada et al. This is an open access article distributed under the Creative Commons Attribution License, which permits unrestricted use, distribution, and reproduction in any medium, provided the original work is properly cited.

The nanocomposites formation by in situ polymerization used a metallocene catalyst (butyl-2-cyclopentadienyl zirconium 2chlorines) and a hectorite synthetic clay type which is discussed. This research was carried out in two phases. The first phase consisted of mixing the components of the metallocenic polymerization reaction (metallocene-methylaluminoxane-ethylene) with clay in a reactor. In the second phase, the metallocenic catalytic system was supported by clay particles and then a polymerization reaction was made. In this second phase, the clay particles were modified using a sol-gel reaction with different $\mathrm{pH}$ values: $\mathrm{pH}=$ $3, \mathrm{pH}=8$, and $\mathrm{pH}=12$. The results were compared in terms of the catalytic activity in the different systems (phase 1 and phase 2) and the nanoparticle morphology of nanocomposites generated in this study.

\section{Introduction}

Inorganic nanoparticles filled in the polymer composites have received increased interest from material scientists. The filler/matrix interface in these nanocomposites might constitute a greater area and hence influence the composites properties to a greater extent with a low filler concentration, in comparison with conventional microparticles composites.

A number of polyolefin nanocomposites based on layered silicates have recently been studied $[1,2]$ and commercialized, such as the poly(propylene)-clay nanocomposites pioneered by General Motors, Basell, and Southern Clay in 2002 [3]. Several methods have been employed to produce polymer nanocomposites with a desired improved mechanical, thermal, optical and gases barriers $\left(\mathrm{O}_{2}, \mathrm{~N}_{2}\right.$, etc. $)$, or other properties. These methods range from simply mixing polymer with fillers to more elaborated approaches such as in situ polymerization [4-10]. A key aspect in the preparation of inorganic/organic nanocomposite materials is the establishment of a good interaction between the polymer matrix and the nanoparticle.
Despite the prime importance of polyolefins in diverse applications, the synthesis of polyolefin-inorganic nanocomposites still remains a scientific challenge because of the poor compatibility of polyolefins with ionic silicates. Although melting intercalation could generate polyolefin nanocomposites $[11,12]$, in the in situ polymerization it has proved to be a promising method for preparing fully exfoliated nanocomposites of polyolefins [13-20]. Tudor et al. [13] reported that a low molecular weight $\mathrm{PP}$ was produced after the methylaluminoxane (MAO) activation of a cationic Ziegler-Natta catalyst. This had been ion-exchanged into the clay interlayer's, but dispersed nanocomposites were not obtained. Bergman et al. [15] intercalated a Brookhart catalyst cationic palladium-based into galleries of an organically modified fluorohectorite and obtained a rubbery polyethylene/clay nanocomposite. More recently, Alexandre et al. [21] prepared PE/clays nanocomposites using a polymerizationfilling technique with a Ti-based geometry catalyst. During the polymerization, a chain transfer agent was added to obtain highly crystalline nanocomposites with improved mechanical properties. 
Recent research studies have been carried out on the catalytic activity during the formation of polyethylene (PE)/clay nanocomposites by in situ polymerization with metallocenes. The modification of the clay with MAO catalyst and the intercalation with octadecylamine (ODA) surfactant were found to play an important role during the in situ formation of PE/clay nanocomposite. ODA-intercalated clay apparently facilitates the activation and monomer insertion processes on zirconocene centers located in internal sites of the clay structure [22].

Nanoparticles are commonly obtained either by the solgel method or by the intercalation method. Sol-gel nanocomposites (polymer/silica nanocomposites) are prepared by in situ hydrolysis and the condensation of mononuclear precursors such as tetraethoxysilane (TEOS) and tetramethoxysilane in organic polymer matrices [23-25]. On the other hand, nanoparticles are obtained by two different methods: The exfoliation of clays or the sol-gel method. Sol-gel nanoparticles are prepared by hydrolysis and condensation of mononuclear precursors such as tetraethoxysilane (TEOS) and tetramethoxysilane in organic polymer matrices [26].

In the literature there is a lot of work which uses sol-gel nanopartículas as a nanofiller [27], but no one has explored the different sol-gel reaction conditions which are aimed to obtain and modify morphology and different characteristics of nanoparticles. During this work the modification of clay was studied using sol-gel reaction at different $\mathrm{pH}$ value. Also the modified clay was used in the obtaining of nanocomposites by in situ polymerization. The present work was divided in two different phases. In the first phase (PHASE I) the components of the reaction, MAO, metallocene, and ethylene, were mixed with clay and the homogeneous polymerization was made. In the second phase (PHASE II), the clay was modified with sol-gel at different $\mathrm{pH}$ values and thereafter the metallocene was supported in the modified clay and the heterogeneous polymerization was made. The activity of the different catalytic system was compared with the dispersion of clay and/or nanoparticles in the nanocomposites obtained.

\section{Experimental Section}

2.1. Materials. The employed clay, synthetic hectorite (HS), was donated by Netherland Organization for Applied Scientific Research-TNO. The characteristics of this clay are described elsewhere [28]. For modification of the clay, tetraethoxy silane (TEOS) of Aldrich and metilaluminoxane (MAO; Witco) were used. The metallocene butyl-2cyclopentadienyl zirconium 2-chlorine was used for ethylene polymerization catalyst which had MAO as the cocatalyst. Ethylene was deoxygenated and dried by passing through columns of $\mathrm{Cu}$ catalyst (BASF) and activated by molecular sieve (13X), respectively. Toluene (solvent) was purified by refluxing, and it was freshly distilled under nitrogen from an $\mathrm{Na}$ /benzophenone system. All manipulations during the catalyst were carried out in an inert nitrogen atmosphere using the Schlenk technique.

\subsection{Methods}

2.2.1. Clay. The clays used in the homogeneous polymerization were used without previous treatment.

2.2.2. Modified Clays. The clay was modified by a sol-gel reaction using tetraethylortosilicate (TEOS) at different $\mathrm{pH}$ values. In a typical procedure, ca. $2.0 \mathrm{~g}$ of clay was dispersed in ethanol and stirred for 10 minutes. Then $\mathrm{HCl}$ or $\mathrm{NH}_{4} \mathrm{OH}$ drops (depending on the $\mathrm{pH}$ value) were added wisely up to reaching $\mathrm{pH}=3.0$ (T3), $\mathrm{pH}=8.0$ (T8), or $\mathrm{pH}=12.0$ (T12). Thereafter, $10 \mathrm{~mL}$ of TEOS was added under inert conditions. After $1 \mathrm{~h}$, the suspension was washed, filtered, and the particles were dried at $200^{\circ} \mathrm{C}$ for a 12 -hour period.

2.2.3. Preparation of the Supported Metallocene. Two grams of modified clay were previously dried with a vacuum for six hours at $130^{\circ} \mathrm{C}$, then the clay particles were dispersed in $30 \mathrm{~mL}$ of purified toluene and placed in contact with $2 \mathrm{~mL}$ of MAO $1.66 \mathrm{~mol} / \mathrm{L}$ for 3 hours with magnetic bar at $70^{\circ} \mathrm{C}$. The slurry was then filtered through a glass filter. The solid was washed several times with toluene at $60^{\circ} \mathrm{C}$ and dried for 4 hours in a vacuum at room temperature. Then an amount of metallocene in solution was added with the metallocene in toluene solution for $3 \mathrm{hrs}$ at $70^{\circ} \mathrm{C}$. The slurry was filtered in a Schlenk equipped with a fritted/filter glass, and washed with toluene. The filtrate was stored and used for the ethylene polymerization. The samples obtained are:

HsT3 = catalytic system supported with modification sol-gel to $\mathrm{pH}=3$.

HsT8 = catalytic system supported with modification sol-gel to $\mathrm{pH}=8$.

HsT12 = catalytic system supported with modification sol-gel to $\mathrm{pH}=12$.

2.2.4. Homopolymerization Reaction. The polymerizations were carried out in a 1-liter Büchi autoclave reactor equipped with a temperature and internal pressure control system with a stirrer. The reactor was coupled to a Brook model $5860 \mathrm{E}$ ethylene flowmeter and connected to data recording software to monitor the consumption of monomer during the course of the reaction.

Catalyst was dissolved in a solvent ( $500 \mathrm{~mL}$ toluene) and it was introduced in the reactor by nitrogen overpressure and the necessary amount of MAO was also added. After evacuating the nitrogen from the reactor, the reaction started by introducing the ethylene until the desired pressure was reached. Reaction time was 30 minutes. The solvent volume was adjusted in such a way that the total volume inside the reactor was $500 \mathrm{~mL}$. The reaction was stopped by the addition of a solution of acidified methanol ( $\mathrm{HCl} 20 \%)$. The polymer was recovered through filtration and washed with methanol and acetone. Finally, the product was dried at room temperature.

Phase 1 (homogeneous polymerization in the presence of clay). The effect of clay addition in the homogeneous polymerization (interaction of clay with MAO and catalyst 
TABLE 1: The addition of component sequences to the catalytic system of polymerization reactions.

\begin{tabular}{ll}
\hline Addition sequence & Code \\
\hline Clay-MAO-catalyst & Hs-MAO-Cat \\
MAO-clay-catalyst & MAO-Hs-Cat \\
MAO-catalyst-clay & MAO-Cat-Hs \\
\hline
\end{tabular}

TABLE 2: The effect of the addition of catalytic system in the homopolymerization reaction.

\begin{tabular}{ll}
\hline Code & Conditions of preparation \\
\hline HsT3 & $\begin{array}{l}\text { Metallocene supported in modification clay using } \\
\text { sol-gel to } \mathrm{pH}=3\end{array}$ \\
HsT8 & $\begin{array}{l}\text { Metallocene supported in modification clays using } \\
\text { sol-gel to } \mathrm{pH}=8\end{array}$ \\
HsT12 & $\begin{array}{l}\text { Metallocene supported in modification clays using } \\
\text { sol-gel to } \mathrm{pH}=2\end{array}$ \\
\hline
\end{tabular}

TABle 3: The effects of the components addition in the reaction characteristics using the catalyst (n-BuCp) ${ }_{2} \mathrm{Zr} \mathrm{Cl}_{2}$.

\begin{tabular}{lccc}
\hline System & $\begin{array}{c}\text { Activity } \\
(\mathrm{kg} \cdot \mathrm{pol} / \mathrm{mol} * \text { bar } * \mathrm{~h})\end{array}$ & $\begin{array}{c}\text { Clay } \\
(\%)\end{array}$ & $\begin{array}{c}\text { Temperature of } \\
\text { reactor }\end{array}$ \\
\hline Homogeneous & 12000 & - & $49-80$ \\
Hs-MAO-Cat & 10210 & 1.2 & $49-80$ \\
MAO-Hs-Cat & 10409 & 1.0 & $49-80$ \\
MAO-Cat-Hs & 9100 & 1.1 & $49-63$ \\
\hline
\end{tabular}

Clay added $(\mathrm{g})=0.32$,

$\mathrm{mol} \mathrm{Zr}$ in the reactor $=3.06 \mathrm{E}-06$,

ratio $\mathrm{Al} / \mathrm{Zr}=1400$,

reaction time (hours) $=0.5$.

in different sequence) was carried out on three different reactions. In all reactions, the ethylene was added at the start to the reactor. After that, the catalytic system component was introduced. The addition of component sequences to the catalytic system of polymerization reactions is shown in the Table 1 . The aim of this study was to determine the condition of the effect in the polymerization reaction.

Phase 2 (heterogeneous polymerization). The reaction conditions were the same as those of the homogenous polymerization.

Three different samples were obtained and are listed in Table 2.

2.3. Characterization. The molecular weights and thier distribution of the produced polyethylene were determined by gel permeation chromatography (GPC) in a Waters Alliance 2000 system equipped with a differential optical refractometer detector. Three separation columns, HT6E, HT5, and HT3 were previously calibrated with narrow molecular weight distribution polystyrene standards which were used. A 1,2,4-trichlorobenzene was used as the solvent. The flow rate for the analysis was $1 \mathrm{~mL} \mathrm{~min}^{-1}$ at $135^{\circ} \mathrm{C}$.

The morphology of the silica nanospheres and its dispersion in the composites were analyzed by transmission electronic microscopy (TEM: JEOL, JEM-1200EXII). Ultrathin specimens with a thickness of about $60 \mathrm{~nm}$ were cut with glass and/or diamond blades by using a cryogenicultramicrotome Leica model EMFCS at $-80^{\circ} \mathrm{C}$.

\section{Results and Discussions}

\subsection{Phase 1}

3.1.1. Establishment of the Reaction Conditions to Obtain Polyethylene Nanocomposites by Means of In Situ Polymerization. The first phase aimed to establish the optimized conditions for obtaining nanocomposites with clay material (1 wt.$\%)$. Besides, it also studied the effects of other components (clay, MAO, and catalysts) in the catalytic activity. With this, the clay percentage in the nanocomposite can be determined. Also characteristics such as morphology and dispersion of the nanoparticles in the nanocomposite were investigated.

3.1.2. The Effects of the Addition of Components in the Polymerization Reaction. Shown in Table 1 is the influence of the addition of component sequences to the catalytic system of polymerization reactions and physical properties of nanocomposites. The results of polymerization reaction are shown in the Table 3, where a low decrease in the catalytic activity for the reactions in which clay was added is observed.

On the other hand, there were not many differences between the HS-MAO-Cat and MAO-HS-Cat systems. With $\mathrm{MAO}-\mathrm{Cat}-\mathrm{Hs}$ system a decrease in the catalytic activity was observed, reaching values of $9100 \mathrm{~kg} \cdot \mathrm{pol} / \mathrm{mol} \mathrm{Zr} * \mathrm{bar} * \mathrm{~h}$. It could be because in Hs-MAO-Cat and MAO-Cat-Hs the interaction between the MAO and the clay was done first, which inactivated the polar groups coming from clay that could deactivate the catalyst. In the MAO-Cat-Hs the clay was added when both (MAO and the catalyst) were in the reaction, slightly deactivating the catalyst.

Through observation of experimental data from the followup of the reaction in the temperature peak, a higher control in the polymerization reaction was found. For the Mao-Cat-Hs system, the temperature ranged between $49^{\circ} \mathrm{C}$ and $63^{\circ} \mathrm{C}$ (T reactor) while for the two other systems, it was between $49^{\circ} \mathrm{C}$ and $80^{\circ} \mathrm{C}$ (similar to the homogeneous reaction). This better control in the polymerization temperature could be due to the active specie stabilization MAO-Cat-Hs, because these two components were added in consecutive order, different to Hs-MAO-Cat and MAO-Hs-Cat systems. The clay percentage found in all the composites obtained was around $1 \%$.

The physical characteristics of the resulting nanocomposites are shown in Table 4. There are no significant variations between the properties for the polymers produced in the presence of clays particles and what was obtained in the homogenous reaction (neat polyethylene without clay). Only the molecular weight $(\mathrm{Mw})$ of the obtained polymers shows a difference according to the catalytic system: for MAOCat-Hs, $\mathrm{Mw}=264 \mathrm{~kg} / \mathrm{mol}$. It is bigger than that found in other systems, even for the homopolymerization reaction. 
TABle 4: Physical characteristics of polyethylene nanocomposites obtained with the three different polymerization systems already studied.

\begin{tabular}{lccccc}
\hline System & $\begin{array}{c}\text { Crystallinity } \\
(\%)\end{array}$ & $\begin{array}{c}\mathrm{Tc} \\
\left({ }^{\circ} \mathrm{C}\right)\end{array}$ & $\begin{array}{c}\mathrm{Tf} \\
\left({ }^{\circ} \mathrm{C}\right)\end{array}$ & $\begin{array}{c}\mathrm{Mw} \\
(\mathrm{kg} / \mathrm{mol})\end{array}$ & $\mathrm{Mw} / \mathrm{Mn}$ \\
\hline Homogenous & 75 & 118 & 135 & 164 & 2.1 \\
Hs-MAO-Cat & 76 & 118 & 135 & 176 & 2.3 \\
MAO-Hs-Cat & 74 & 118 & 134 & 167 & 2.3 \\
MAO-Cat-Hs & 72 & 118 & 133 & 264 & 2.4 \\
\hline
\end{tabular}

This is in agreement with the highest control of the reaction temperature $\left(49^{\circ} \mathrm{C}-63^{\circ} \mathrm{C}\right)$ achieved for this system.

This better control of temperature might have minimized chain transfer and the beta elimination reactions, which are responsible for decreasing molecular weight during polymerization reaction. Apparently, for Hs-MAO-Cat and MAO-Hs-Cat systems, the behavior of the supported catalyst system is similar to that described by Tait: a bidimensional liquid, which favors the mobility of the active species. Which in turn produces polymers with characteristics closer to those obtained with the homogeneous systems [29]. It is worth noting that the supported species are indeed different in the third case, which seems as if it is really acting as a heterogeneous catalytic: species are immobilized and there is a lower probability of biomolecular deactivation reactions. Therefore polymer molecular weight increases [30]. To sum up, MAO-Cat-Hs showed the best conditions for the best control in the reaction and produced polymers of higher molecular weight. Therefore, in the next stage this system was further exploited. The melting and Crystallinity temperature of the nanocomposites did not significantly change compared to the neat $\mathrm{PE}$, indicating the formation of linear polyethylene.

\subsection{Phase 2}

3.2.1. The Effects of Clay Modification by Means of the Sol-Gel Method with Different $p H$ Values and Their Use as a Supported Metallocene System. Clay modifications were carried out by means of sol-gel synthesis at different $\mathrm{pH}$ values in order to identify its effect on the particle morphology during the sol-gel reaction as well as in the properties of resulting nanocomposites.

It is known that the sol-gel method allows the morphology manipulation of the particles as well as its size, depending on the $\mathrm{pH}$ value in which the synthesis is carried out $[25,31-33]$ as follows: for $\mathrm{pH}$ basic values $(\mathrm{pH}=12)$ spherical morphologies are formed; where $\mathrm{pH}$ acid fibrillar morphologies $(\mathrm{pH}=3)$ are obtained. With $\mathrm{pH}$ near to neuter values it is preferred to form morphologies in nets with fibrillar characteristics $(\mathrm{pH}=8)$.

This modification of clay characteristics is carried out in order to increase its interlaminar space (due to the formation of these compounds within the interlaminar region) in order to easily render the dispersion of the nanosheets within the polymer. Furthermore, the influence of the particles morphology in the MAO-metallocene systems was investigated.
Three new systems were established for this phase by supporting the metallocene catalysts in the modified clay at different $\mathrm{pH}$ values, the samples are listed in Table 2 .

Table 5 shows the reaction conditions established for the modified clay by means of sol-gel reaction at different $\mathrm{pH}$ values. The impregnation reactions of the catalyst in the different systems were done with similar amounts $(0.72 \mathrm{~g})$. The quantity of supported catalytic and experimentally obtained were more effiecient for $\mathrm{pH}$ values of 3 and 8 than for a $\mathrm{pH}$ value of 12 . When the modification of $\mathrm{pH}=12$ was used, a lower catalyst loading into the modified clay was achieved. This smaller incorporation could be due to the reduced number of $\mathrm{OH}$ groups resulting from the treatment with alkaline $\mathrm{pH}$. One cannot neglect that different texture properties may also affect further research necessary to clarify this point.

On the other hand, for all the three cases, a considerable decrease in catalyst activity was observed when it was supported in modified clay by sol-gel reaction. Comparing it with both, the homopolymerization and the heterogeneous reactions (when the clay was added directly to the reactor without supporting it), the highest decrease in catalytic activity was observed in the case of HsT8 (up to 84\%). For HsT3 and HsT12, the decrease in catalyst activity is low compared to the HsT8 system: its value is roughly one half of that obtained in the case of homopolymerization reaction. This decrease effect of catalytic activity with the clay support has been widely studied [34]. It is probably due to the clay surface which should work like an extremely huge ligand, proving more difficult to render access to the monomer.

The catalytic systems, which involved extreme $\mathrm{pH}$ values, that is, HsT3 ( $\mathrm{pH}=3)$ and HsT12 ( $\mathrm{pH}=12)$, showed higher catalyst activity in comparison to that carried out with HsT8 system. This behavior can be attributed to the potential generation of a higher $\mathrm{OH}$ density on the clay surface (when high basic or acid $\mathrm{pH}$ value are used, a higher amount of $\mathrm{OH}$ group are formed) which acts as capacitor plate, depending on the $\mathrm{pH}$ media.

The higher the $\mathrm{OH}$ density, the better it can afford a better interaction between the clay surface and MAO, which in turn allows the metallocene catalytic to be a more positive load in their active center and also to present an increase in the catalytic activity. Comparing this with the HsT8 system $(\mathrm{pH}=8)$, which does not present a big formation of $\mathrm{OH}$ sites, therefore the MAO stays chemically more available to interact with catalytic and to decrease the activity as well.

It was observed that the modification with the sol-gel method in different $\mathrm{pH}$ values has a big influence on the metallocene catalytic system. It was obtained that acid $\mathrm{pH}$ values $(\mathrm{pH}=3)$ and basic $(\mathrm{pH}=12)$ show greater activity values compared to intermediate $\mathrm{pH}$ values $(\mathrm{pH}=8)$. As one expects, this behavior (that the nanoparticle morphology depending on the $\mathrm{pH}$ ) could have big repercussions in the mechanical properties of the obtained nanocomposites.

3.2.2. Transmission Electronic Microscopy. Figure 1 shows the TEM images of the different obtained nanocomposites. It can be appreciated that for the two phases, it found a good dispersion of nanoparticles in the polyethylene matrix and 
TABLE 5: The effects of modification to clay using sol-gel, in the reaction conditions of polyethylene nanocomposites.

\begin{tabular}{lcccc}
\hline System & System $(\mathrm{g})$ & $\begin{array}{c}\% \mathrm{Zr} \\
(\text { experimental })\end{array}$ & $\begin{array}{c}\text { Activity } \\
(\mathrm{kg} \cdot \mathrm{pol} / \mathrm{mol} * \mathrm{bar} * \mathrm{~h})\end{array}$ & $\begin{array}{c}\text { Clay \% } \\
\text { Homogeneous }\end{array}$ \\
HsT3 & - & - & 5142 & - \\
HsT8 & 0.044 & 0,64 & 1901 & 0.3 \\
HsT12 & 0.046 & 0,64 & 5250 & 0.8 \\
\hline
\end{tabular}

Catalytic system $(\mathrm{g})=0.048$,

$\% \mathrm{Zr}$ theoretical $=0.72$,

$\mathrm{mol} \mathrm{Zr}$ in reactor $=3.08 E-06$,

ratio $\mathrm{Al} / \mathrm{Zr}=1400$,

time of reaction $(\mathrm{h})=0.5$.

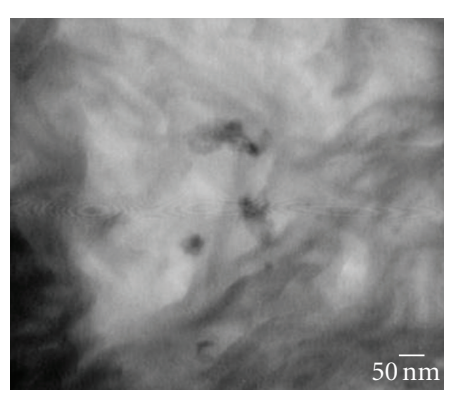

HS-MAO-Cat

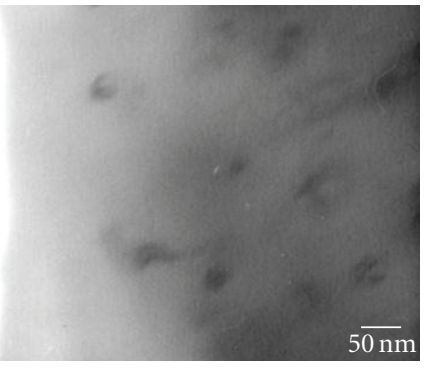

HsT3

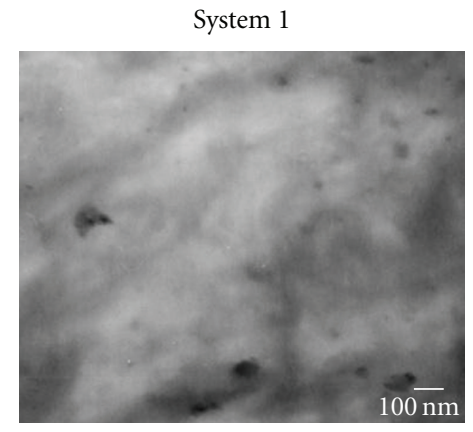

MAO-Hs-Cat

System 2

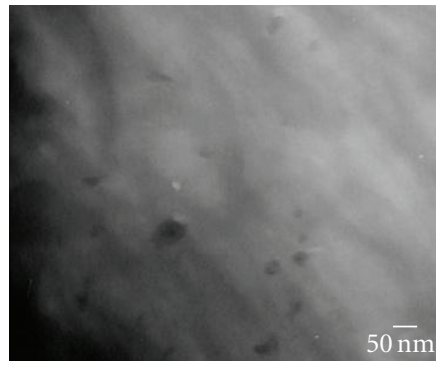

HsT8

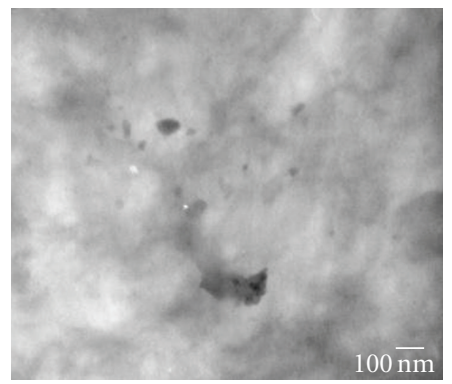

MAO-Cat-Hs

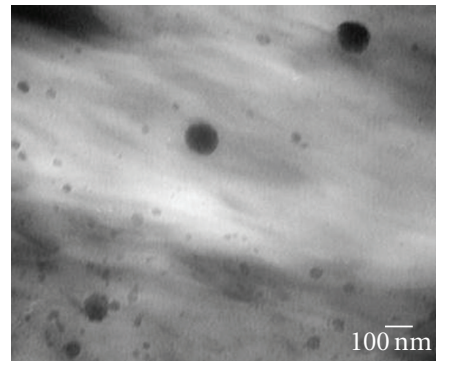

HsT12

FIgURE 1: Transmission electronic microscopy images of different obtained nanocomposites.

various morphology nanoparticles were discovered. This could be because the clay used was synthetic and it could suffer a breaking of the laminated structure that changes the morphologies after of modification with sol-gel reaction.

In Figure 1 the first phase presents agglomerations of nanoparticles. Only in the MAO-Cat-Hs system is it possible to see agglomeration with greater size particles to $100 \mathrm{~nm}$ (nanoparticles of sizes lower than $100 \mathrm{~nm}$ and aggregates of approximately $200 \mathrm{~nm}$ ); this system did not get a total exfoliation. For the two other studied systems, different sizes in nanoparticles were principally found, but all of them were lower than $100 \mathrm{~nm}$ in the polymeric matrix. What is mentioned above allows it to establish an adequate nanocomposites formation for the three studied systems.

In Figure 1 the second phase shows different morphologies of particles depending on $\mathrm{pH}$ value to which the clays were modified.
The particles modified with $\mathrm{pH}=3$ (with this condition of sol-gel reaction, fiber is the preferable morphology) can be observed in small aggregates with 5-10 sheets or fibers dispersed in the polymer. The size of these aggregates average from $20 \mathrm{~mm}$ in thickness and the length can reach $70 \mathrm{~mm}$.

When the clay modification was carried out to a $\mathrm{pH}=8$ it was observed that the particles morphology, its distributions, and its size were similar to the results obtained in phase 1, showing nanoparticles dispersed in the polymer.

When the clay modification was at a $\mathrm{pH}=12$, it was possible to observe a preferably spherical morphology, which is normal in the morphology that was obtained for the sol-gel reaction at basic $\mathrm{pH}$ values. The different size particles were obtained from $5 \mathrm{~nm}$ to approximately $100 \mathrm{~nm}$.

Evidently, the clay particles adopted a particle morphology generated by the sol-gel reaction; this would imply very strong sol-gel reaction conditions to be supported by 
synthetic clay as the case in this research. Due to this fibrillates, layered, or spherical morphologies can be obtained, depending on the conditions of modification. On the other hand, smaller-sized particles were obtained through clay modifications with the sol-gel method, without taking into account the $\mathrm{pH}$ value used to compare it with the clay addition to the catalytic system.

\section{Conclusion}

The modifications of the layer of clays using the sol-gel technique at different $\mathrm{pH}$ values present new characteristics for the modification of the clays.

The clays modified with sol-gel technique at different $\mathrm{pH}$ values, present big modifications in the morphology, when used as catalytic support in the obtention of in situ polyolefins nanocomposites.

The catalytic activity presents large increases when the lays was modified with sol-gel reaction in acidic or a basic condition compared with neutral condition and with other works of the literature.

Best dispersion of nanoparticles is obtained when the clays are modified with sol-gel technique in the obtention of in situ nanocomposites.

\section{Acknowledgments}

The authors acknowledge the financial support of CONICYT under Projects FONDAP 11980002 and Fondecyt 1050651. The supply of commercial polypropylene clays by Dr. Hartmut Fischer from the Netherland Organization for Applied Scientific Research-TNO, is gratefully acknowledged. Special thanks are to Professor Dr. Gerhard Wegner and Dr. Ingo Lieberwirth of the Max Planck Institute for Polymer Research, Mainz, Germany, for providing the TEM equipment. E. Moncada thanks Professor Dr. João Henrique dos Santos of Universidade Federal do Rio Grande do Sul, Porto Alegre, Brazil, for the collaboration in the discussion of the result, and the Deutscher Akademischer Austauschdienst (DAAD) for a Ph.D. fellowship.

\section{References}

[1] E. P. Giannelis, "Polymer layered silicate nanocomposites," Advanced Materials, vol. 8, no. 1, pp. 29-35, 1996.

[2] R. A. Vaia, K. D. Jandt, E. J. Kramer, and E. P. Giannelis, "Microstructural evolution of melt intercalated polymerorganically modified layered silicates nanocomposites," Chemistry of Materials, vol. 8, no. 11, pp. 2628-2635, 1996.

[3] R. Stewart, "Nanocomposites: microscopic reinforcements boost polymer performance," Plastics Engineering, vol. 60, no. 5, pp. 22-29, 2004.

[4] P. Judeinstein and C. Sanchez, "Hybrid organic-inorganic materials: a land of multidisciplinarity," Journal of Materials Chemistry, vol. 6, no. 4, pp. 511-525, 1996.

[5] G. Kickelbick, "Concepts for the incorporation of inorganic building blocks into organic polymers on a nanoscale," Progress in Polymer Science, vol. 28, no. 1, pp. 83-114, 2003.
[6] N. Y. Kim, N. L. Jeon, I. S. Choi et al., "Surface-initiated ring-opening metathesis polymerization on $\mathrm{Si} / \mathrm{SiO}_{2}$," Macromolecules, vol. 33, no. 8, pp. 2793-2795, 2000.

[7] C. M. Chan, J. Wu, J. X. Li, and Y. K. Cheung, "Polypropylene/calcium carbonate nanocomposites," Polymer, vol. 43, no. 10, pp. 2981-2992, 2002.

[8] R. C. Advincula, "Surface initiated polymerization from nanoparticle surfaces," Journal of Dispersion Science and Technology, vol. 24, no. 3-4, pp. 343-361, 2003.

[9] Y. M. Cao, J. Sun, and D. H. Yu, "Swelling kinetics of poly(Nvinylimidazole-co-sodium styrenesulfonate) hydrogels," Journal of Applied Polymer Science, vol. 83, no. 1, pp. 191-200, 2002.

[10] M. W. Weimer, H. Chen, E. P. Giannelis, and D. Y. Sogah, "Direct synthesis of dispersed nanocomposites by in situ living free radical polymerization using a silicate-anchored initiator," Journal of the American Chemical Society, vol. 121, no. 7, pp. 1615-1616, 1999.

[11] M. Kawasumi, N. Hasegawa, M. Kato, A. Usuki, and A. Okada, "Preparation and mechanical properties of polypropylene-clay hybrids," Macromolecules, vol. 30, no. 20, pp. 6333-6338, 1997.

[12] H. G. Jeon, H. T. Jung, S. W. Lee, and S. D. Hudson, "Morphology of polymer/silicate nanocomposites: high density polyethylene and a nitrile copolymer," Polymer Bulletin, vol. 41, no. 1, pp. 107-113, 1998.

[13] J. Tudor, L. Willington, D. O'Hare, and B. Royan, "Intercalation of catalytically active metal complexes in phyllosilicates and their application as propene polymerisation catalysts," Chemical Communications, no. 17, pp. 2031-2032, 1996.

[14] J. Tudor and D. O’Hare, “Stereospecific propene polymerisation catalysis using an organometallic modified mesoporous silicate," Chemical Communications, no. 6, pp. 603-604, 1997.

[15] J. S. Bergman, H. Chen, E. P. Giannelis, M. G. Thomas, and G. W. Coates, "Synthesis and characterization of polyolefinsilicate nanocomposites: a catalyst intercalation and in situ polymerization approach," Chemical Communications, no. 21, pp. 2179-2180, 1999.

[16] J. Heinemann, P. Reichert, R. Thomann, and R. Mülhaupt, "Polyolefin nanocomposites formed by melt compounding and transition metal catalyzed ethene homo- and copolymerization in the presence of layered silicates," Macromolecular Rapid Communications, vol. 20, no. 8, pp. 423-430, 1999.

[17] M. Alexandre, P. Dubois, R. Jerome et al., "Polyolefin nanocomposites," US Patent, no. WO 9947598, 1999.

[18] J. Wang, Z. Liu, C. Guo, Y. Chen, and D. Wang, "Preparation of a PE/MT composite by copolymerization of ethylene with in-situ produced ethylene oligomers under a dual functional catalyst system intercalated into MT layer," Macromolecular Rapid Communications, vol. 22, no. 17, pp. 1422-1426, 2001.

[19] Y. H. Jin, H. J. Park, S. S. Im, S. Y. Kwak, and S. Kwak, "Polyethylene/clay nanocomposite by in-situ exfoliation of montmorillonite during Ziegler-Natta polymerization of ethylene," Macromolecular Rapid Communications, vol. 23, no. 2, pp. 135-140, 2002.

[20] Q. Wang, Z. Zhou, L. Song, H. Xu, and L. Wang, "Nanoscopic confinement effects on ethylene polymerization by intercalated silicate with metallocene catalyst," Journal of Polymer Science A, vol. 42, no. 1, pp. 38-43, 2004.

[21] M. Alexandre, P. Dubois, T. Sun, J. M. Garces, and R. Geronime, "Polyethylene-layered silicate nanocomposites prepared by the polymerization-filling technique: synthesis and mechanical properties," Polymer, vol. 43, no. 8, pp. 2123-2132, 2002. 
[22] P. Zapata, R. Quijada, C. Covarrubias, E. Moncada, and J. Retuert, "Catalytic activity during the preparation of $\mathrm{PE} /$ clay nanocomposites by in situ polymerization with metallocene catalysts," Journal of Applied Polymer Science, vol. 113, no. 4, pp. 2368-2377, 2009.

[23] H. P. Lin and M. Chung-Yuan, "Structural and morphological control of cationic surfactant-templated mesoporous silica," Accounts of Chemical Research, vol. 35, no. 11, pp. 927-935, 2002.

[24] W. K. Dong, A. Blumstein, J. Kumar, and S. Tripathy, "Layered Aluminosilicate/Chromophore Nanocomposites and Their Electrostatic Layer-by-Layer Assembly," Chemistry of Materials, vol. 13, pp. 243-246, 2001.

[25] G. J. D. A. A. Soler-Illia, C. Sanchez, B. Lebeau, and J. Patarin, "Chemical strategies to design textured materials: from microporous and mesoporous oxides to nanonetworks and hierarchical structures," Chemical Reviews, vol. 102, no. 11, pp. 4093-4138, 2002.

[26] W. Liangming, T. Tao, and H . Baotong, "Synthesis and characteization of polyethylene/clay-silica nanocomposites: a montmorillonite/silica-hybrid-supported catalyst and in situ polymerization," Journal of Polymer Science A, vol. 42, no. 4, pp. 941-949, 2004.

[27] P. Zapata, R. Quijada, and R. Benavente, "In situ formation of nanocomposites based on polyethylene and silica nanospheres," Journal of Applied Polymer Science, vol. 119, no. 3, pp. 1771-1780, 2011.

[28] E. Moncada, R. Quijada, and J. Retuert, "Comparative effect of metallocene and Ziegler-Natta polypropylene on the exfoliation of montmorillonite and hectorite clays to obtain nanocomposites," Journal of Applied Polymer Science, vol. 103, no. 2, pp. 698-706, 2007.

[29] A. M. Johannes and J. T. Peter, "The "comonomer effect" in ethylene/a-olefin copolymerization using homogeneous and silica-supported $\mathrm{Cp} 2 \mathrm{ZrCl} 2 / \mathrm{MAO}$ catalyst systems: Some insights from the kineticsof polymerization, active center studies, and polymerization temperature," Journal of Polymer Science A, vol. 46, no. 1, pp. 267-277, 2007.

[30] W. Kaminsky and F. Renner, "High melting polypropenes by silicasupported zirconocene catalysts," Die Makromolekulare Chemie, Rapid Communications, vol. 14, no. 4, p. 239, 1993.

[31] E. Moncada, R. Quijada, and J. Retuert, "Nanoparticles prepared by the sol-gel method and their use in the formation of nanocomposites with polypropylene," Nanotechnology, vol. 18, no. 33, Article ID 335606, 2007.

[32] M. Ogawa, "Preparation of layered silicadialkyldimethylammonium bromide nanocomposites," Langmuir, vol. 13, no. 6, pp. 1853-1855, 1997.

[33] S. K. Yun and J. Maier, "Layered and hexagonal aluminosilicate-hexadecylamine mesostructures: solid state transformation and ionic conductivity," Inorganic Chemistry, vol. 38, no. 3, pp. 545-549, 1999.

[34] F. C. Franceschini, T. T. D. R. Tavares, J. H. Z. Dos Santos, M. L. Ferreira, and J. B. P. Soares, "Ethylene and propylene polymerization using in situ supported $\mathrm{Me}_{2} \mathrm{Si}$ (Ind) ${ }_{2} \mathrm{ZrCl}_{2}$ Catalyst: experimental and theoretical study," Macromolecular Materials and Engineering, vol. 291, no. 3, pp. 279-287, 2006. 

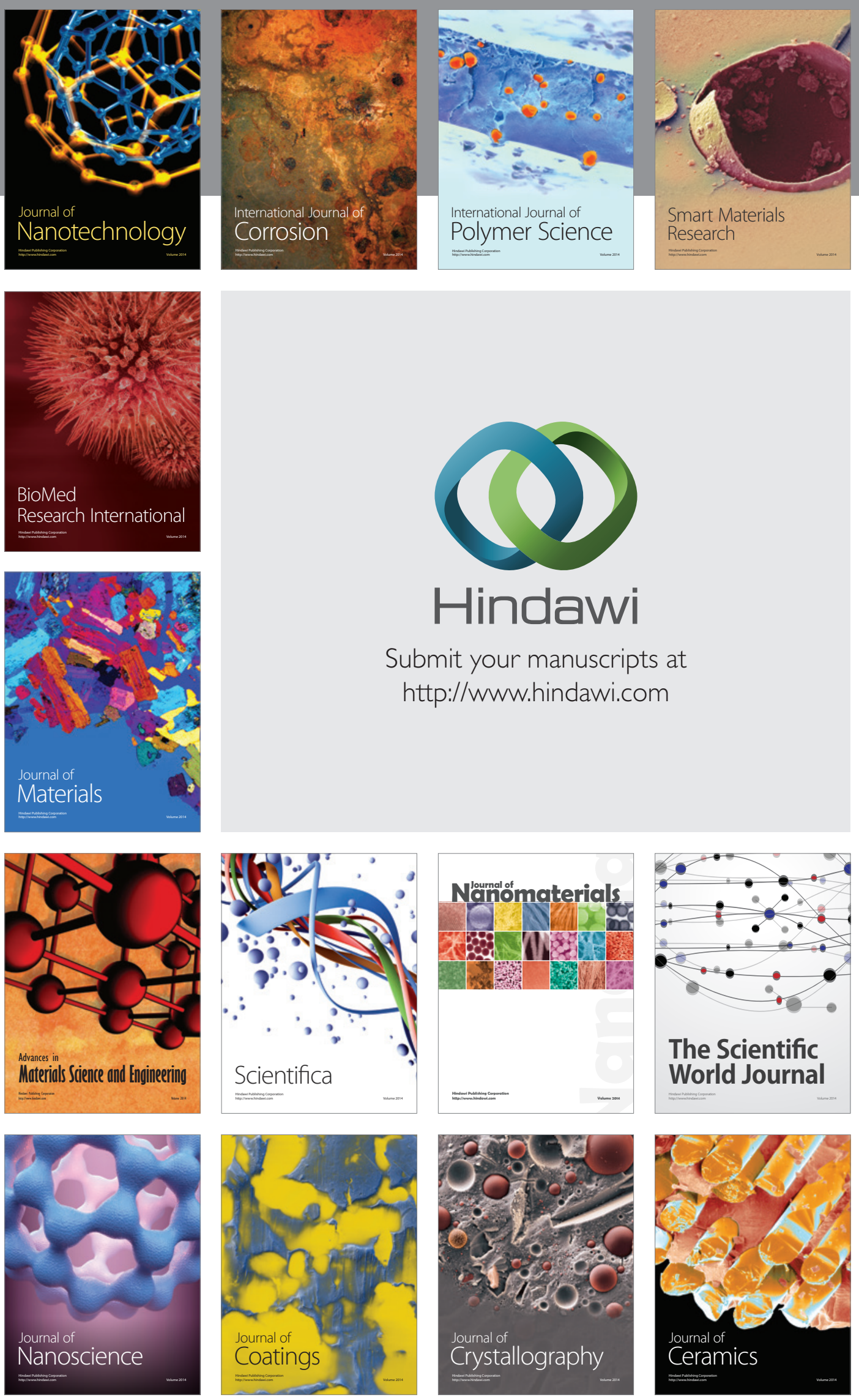

The Scientific World Journal

Submit your manuscripts at

http://www.hindawi.com

\section{World Journal}

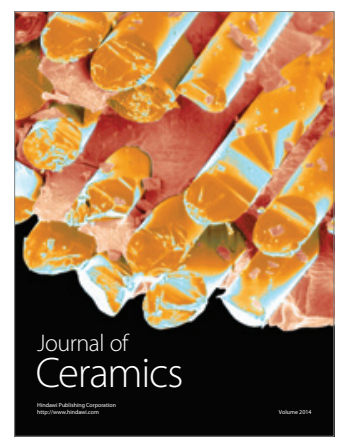

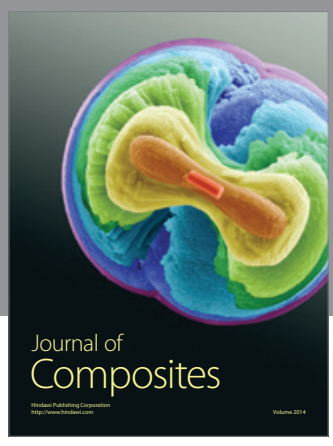
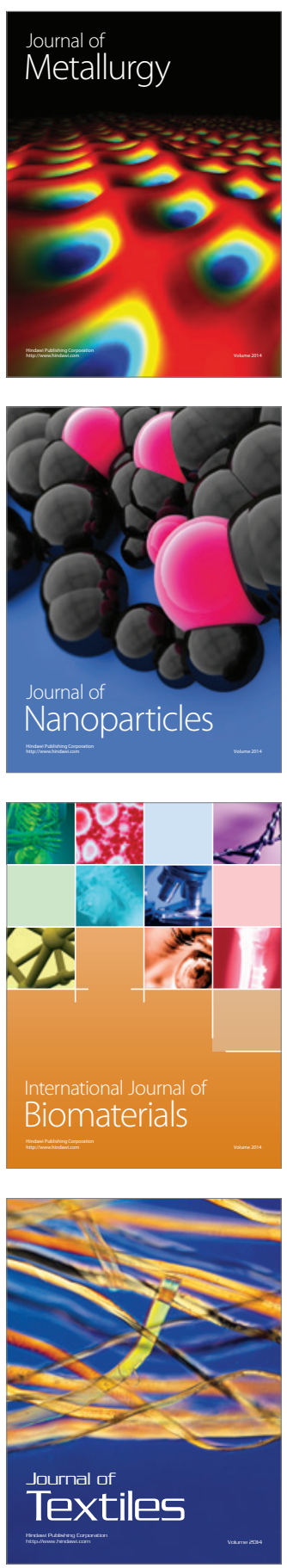